\title{
Tumor Response Domain
}

National Cancer Institute

\section{Source}

National Cancer Institute. Tumor Response Domain. NCI Thesaurus. Code C102722.

A subject domain utilized for the submission of information encompassing and representing data, vocabulary or records related to tumor response. 